\title{
On the Page Number of Upward Planar Directed Acyclic Graphs ${ }^{\star}$
}

\author{
Fabrizio Frati $^{1,2,3}$, Radoslav Fulek ${ }^{1}$, and Andres J. Ruiz-Vargas ${ }^{1}$ \\ 1 School of Basic Sciences - École Polytechnique Fédérale de Lausanne, Switzerland \\ \{fabrizio.frati, radoslav.fulek, andres.ruizvargas\} @epfl.ch \\ 2 Dipartimento di Informatica e Automazione, Università Roma Tre \\ 3 School of Information Technologies, University of Sydney
}

\begin{abstract}
In this paper we study the page number of upward planar directed acyclic graphs. We prove that: (1) the page number of any $n$-vertex upward planar triangulation $G$ whose every maximal 4 -connected component has page number $k$ is at most $\min \left\{O(k \log n), O\left(2^{k}\right)\right\}$; (2) every upward planar triangulation $G$ with $o\left(\frac{n}{\log n}\right)$ diameter has $o(n)$ page number; and (3) every upward planar triangulation has a vertex ordering with $o(n)$ page number if and only if every upward planar triangulation whose maximum degree is $O(\sqrt{n})$ does.
\end{abstract}

\section{Introduction}

A $k$-page book embedding of a graph $G=(V, E)$ is a total ordering $\sigma$ of $V$ and a partition of $E$ into subsets $E_{1}, E_{2}, \ldots, E_{k}$, called pages, such that no two edges $(u, v)$ and $(w, z)$ with $u<_{\sigma} w<_{\sigma} v<_{\sigma} z$ belong to the same set $E_{i}$. The page number of $G$ is the minimum $k$ such that $G$ admits a $k$-page book embedding.

Book embeddings (first introduced by Kainen [15] and by Ollmann [19]) find applications in several contexts, such as VLSI design, fault-tolerant processing, sorting networks, and parallel matrix multiplication (see, e.g., [4|1120]21]). Henceforth, they have been widely studied from a theoretical point of view; namely, the literature is rich of combinatorial and algorithmic contributions on the page number of various classes of graphs (see, e.g., [2|7|8|9|10|17|18]). We remark here a famous result of Yannakakis [22] stating that any planar graph has page number at most four.

Heath et al. [13 14] extended the notions of book embedding and page number to directed acyclic graphs ( $D A G s$ for short) in a very natural way: Given a DAG $G=(V, E)$, book embedding and page number of $G$ are defined as for undirected graphs, except that the total ordering of $V$ is now required to be a linear extension of the partial order of $V$ induced by $E$. That is, if $G$ contains an edge from a vertex $u$ to a vertex $v$, then $u<_{\sigma} v$ in any feasible total ordering $\sigma$ of $V$. The authors of [13]14] showed that DAGs with page number equal to one can be characterized and recognized efficiently; however, they proved that, in general, determining the page number of a DAG is NP-complete.

\footnotetext{
* Work partially supported by the Italian Ministry of Research, grant RBIP06BZW8, FIRB project "Advanced tracking system in intermodal freight transportation", by the Swiss National Science Foundation 200021-125287/1, by the ESF project 10-EuroGIGA-OP-003 "Graph Drawings and Representations", and by the MIUR of Italy, project AlgoDEEP 2008TFBWL4.
} 
The main problem raised by Heath et al. and studied in, e.g., [16 12[13]14], is whether every upward planar DAG admits a book embedding in few pages. An upward planar DAG is a DAG that admits a drawing which is simultaneously upward, i.e., each edge is represented by a curve monotonically increasing in the $y$-direction, and planar, i.e., no two edges cross. Upward planar DAGs are the natural counterpart of planar graphs in the context of directed graphs. Notice that there exist DAGs which admit a planar non-upward embedding and that require $\Omega(|V|)$ pages in any book embedding [12|14]. No upper bound better than the trivial $O(|V|)$ and no lower bound better than the trivial $\Omega(1)$ are known for the page number of upward planar DAGs. It is however known that directed trees have page number one [14], that unicyclic DAGs have page number two [14], and that series-parallel DAGs have page number two [16].

In this paper we study the page number of upward planar DAGs. Before stating our results we need some background.

First, it is known that every upward planar DAG $G$ can be augmented to an upward planar triangulation $G^{\prime}$ [5]. That is, edges can be added to $G$ so that the resulting graph $G^{\prime}$ is still an upward planar DAG and every face of $G^{\prime}$ is delimited by a 3-cycle. Thus, in order to establish tight bounds on the page number of upward planar DAGs, it suffices to look at upward planar triangulations, as the page number of a subgraph $G$ of a graph $G^{\prime}$ is at most the page number of $G^{\prime}$. In the following, unless otherwise specified, all the considered graphs are upward planar triangulations.

Second, consider a total ordering $\sigma$ of $V$. A twist is a set of pairwise crossing edges, i.e., a set $\left\{\left(u_{1}, v_{1}\right),\left(u_{2}, v_{2}\right), \ldots,\left(u_{k}, v_{k}\right)\right\}$ of edges such that $u_{1}<_{\sigma} u_{2}<_{\sigma} \cdots<_{\sigma} u_{k}<_{\sigma}$ $v_{1}<{ }_{\sigma} v_{2}<{ }_{\sigma} \cdots<_{\sigma} v_{k}$. It is straightforward that the page number of a graph $G$ is lower bounded by the minimum over all vertex orderings $\sigma$ of the maximum size of a twist in $\sigma$. Moreover, a function of the maximum size of a twist in a vertex ordering upper bounds the page number of an $n$-vertex graph $G$, as stated in the following two lemmata.

Lemma 1. [3] Let $\sigma$ be a vertex ordering of an n-vertex graph $G$. Suppose that the maximum twist of $\sigma$ has size $k$. Then $G$ admits a book embedding with vertex ordering $\sigma$ and with $O(k \log n)$ pages.

Lemma 2. [16] Let $\sigma$ be a vertex ordering of an $n$-vertex graph $G$. Suppose that the maximum twist of $\sigma$ has size $k$. Then $G$ admits a book embedding with vertex ordering $\sigma$ and with $O\left(2^{k}\right)$ pages.

Thus, in order to get upper bounds for the page number of a graph, it often suffices to construct vertex orderings with small maximum twist size.

In this paper we consider the relationship between the page number of an $n$-vertex upward planar triangulation $G$ and three important graph parameters of $G$ : The connectivity, the diameter, and the degree. We show the following results. (i) In Sect. 3, we prove that an upward planar triangulation $G$ admits a vertex ordering with maximum twist size $O(f(n))$ if and only if every maximal 4 -connected component of $G$ does. As a corollary, upward planar 3-trees have constant page number. (ii) In Sect. 4, we prove that every upward planar triangulation $G$ has a vertex ordering whose maximum twist size is a function of the diameter of $G$, that is, of the length of the longest directed path in $G$. As a corollary, every upward planar triangulation whose diameter is $o(n / \log n)$ 
admits a book embedding in $o(n)$ pages. (iii) In Sect. 5. we show that every upward planar triangulation has a vertex ordering with $o(n)$ page number if and only if every upward planar triangulation whose maximum degree is $O(\sqrt{n})$ does.

\section{Definitions}

A directed graph is a graph with direction on the edges. The underlying graph of a directed graph $G$ is the undirected graph obtained from $G$ by removing the directions on its edges. We denote by $(u, v)$ an edge directed from a vertex $u$, which is called the origin of $(u, v)$, to a vertex $v$, which is called the destination of $(u, v)$; edge $(u, v)$ is incoming $v$ and outgoing $u$. A source (resp. sink) is a vertex with no incoming edge (resp. with no outgoing edge). A directed cycle is a directed graph whose underlying graph is a cycle and containing no source and no sink. A directed acyclic graph (DAG for short) is a directed graph containing no directed cycle. A directed path is a directed graph whose underlying graph is a path and containing exactly one source and one sink. The diameter of a directed graph is the number of vertices in its longest directed path.

A drawing of a directed graph is a mapping of each vertex to a point in the plane and of each edge to a Jordan curve between its end-points. A drawing is upward if each edge $(u, v)$ is a curve monotonically increasing in the $y$-direction and it is planar if no two edges intersect except, possibly, at common end-points. A drawing is upward planar if it is both upward and planar. An upward planar graph is a graph that admits an upward planar drawing. A planar drawing of a graph partitions the plane into connected regions, called faces. The unbounded face is the outer face, all the other faces are internal faces. Two upward planar drawings of an upward planar DAG are equivalent if they determine the same clockwise ordering of the edges around each vertex. An embedding of an upward planar DAG is an equivalence class of upward planar drawings. An embedded upward planar graph is an upward planar DAG together with an embedding.

An upward planar triangulation is an upward planar graph whose underlying graph is a maximal planar graph. Consider any two upward planar drawings $\Gamma_{1}$ and $\Gamma_{2}$ of an upward planar triangulation $G$. Then, either $\Gamma_{1}$ and $\Gamma_{2}$ are equivalent, or the clockwise ordering of the edges around each vertex in $\Gamma_{1}$ is exactly the opposite of the one in $\Gamma_{2}$. The outer face of an upward planar drawing $\Gamma$ of an upward planar triangulation $G$ is delimited by a cycle composed of three edges $(u, v),(u, z)$, and $(v, z)$. Then, $u, v$, and $z$ are called bottom vertex, middle vertex, and top vertex of $\Gamma$, respectively. Consider the two embeddings $\mathcal{E}_{1}$ and $\mathcal{E}_{2}$ of an upward planar triangulation $G$. Then, the bottom, middle, and top vertex of $\mathcal{E}_{1}$ coincide with the bottom, middle, and top vertex of $\mathcal{E}_{2}$, respectively. Hence such vertices are simply called the bottom vertex of $G$, the middle vertex of $G$, and the top vertex of $G$, respectively.

A total vertex ordering $\sigma$ of a DAG $G$ is upward if $G$ has no edge $(u, v)$ such that $v<_{\sigma} u$. The upward vertex orderings are all and only the vertex orderings that are feasible for a book embedding of a DAG. We say that an upward vertex ordering $\sigma$ induces a twist of size $k$ if $G$ contains edges $\left(u_{1}, v_{1}\right), \ldots,\left(u_{k}, v_{k}\right)$ such that $u_{1}<_{\sigma} \ldots<_{\sigma} u_{k}<_{\sigma} v_{1}<_{\sigma} \ldots, v_{k}$. The maximum twist size of an upward vertex ordering $\sigma$ is the maximum number of edges in a twist induced by $\sigma$. Two edges $\left(u_{1}, v_{1}\right)$ and $\left(u_{2}, v_{2}\right)$ are nested in $\sigma$ if $u_{1}<_{\sigma} u_{2}<_{\sigma} v_{2}<_{\sigma} v_{1}$. Two edges $\left(u_{1}, v_{1}\right)$ and $\left(u_{2}, v_{2}\right)$ cross in $\sigma$ if $u_{1}<_{\sigma} u_{2}<_{\sigma} v_{1}<_{\sigma} v_{2}$. 
An undirected graph is $k$-connected if the removal of any $k-1$ vertices leaves the graph connected. A directed graph is $k$-connected if its underlying graph is. A maximal $k$-connected component of a graph $G$ is a subgraph $G^{\prime}$ of $G$ such that $G^{\prime}$ is $k$-connected and no subgraph $G^{\prime \prime}$ of $G$ with $G^{\prime} \subset G^{\prime \prime}$ is $k$-connected. A separating triangle $C$ in a graph $G$ is a 3-cycle such that the removal of the vertices of $C$ from $G$ disconnects $G$. A separating triangle $C$ in a graph $G$ is maximal if $G$ has no separating triangle $C^{\prime}$ such that $C$ is internal to $C^{\prime}$.

The degree of a vertex is the number of edges incident to it. The degree of a graph is the maximum among the degrees of its vertices. A DAG is Hamiltonian if it contains a directed path passing through all its vertices. An Hamiltonian DAG $G$ has exactly one upward total vertex ordering. Moreover, if $G$ is upward planar, then it has page number at most 2. A plane 3-tree is a maximal plane graph that can be constructed as follows. Let $G_{3}$ be a 3 -cycle embedded in the plane. A plane 3 -tree with $n$ vertices is a plane graph that can be constructed from a plane graph $G_{n-1}$ with $n-1$ vertices by inserting a vertex inside an internal face of $G_{n-1}$ and by connecting such a vertex to the three vertices incident to the face. A planar 3-tree is a planar graph that can be embedded as a plane 3-tree. An upward plane 3-tree is an upward planar DAG whose underlying graph is a plane 3 -tree.

\section{Page Number and Connectivity}

In this section we study the relationship between the page number of an upward planar DAG and the page number of its maximal 4-connected components. We prove the following:

Theorem 1. Let $f(n)$ be any function such that $f(n) \in \Omega(1)$ and $f(n) \in O(n)$. Consider any $n$-vertex upward planar triangulation $G$ and suppose that every maximal 4-connected component of $G$ has an upward vertex ordering with maximum twist size at most $f(n)$. Then $G$ has an upward vertex ordering with maximum twist size $O(f(n))$.

First, we define a rooted tree $T=\left(V^{\prime}, E^{\prime}\right)$, whose nodes correspond to subgraphs of $G=(V, E)$, which reflects the structure of separating triangles in $G$. Tree $T$ is recursively defined as follows (see Fig. 1(a) . The root $r$ of $T$ corresponds to $G^{\prime}(r)=G$. Suppose that a node $a$ of $T$ corresponds to a subgraph $G^{\prime}(a)$ of $G$. If $G^{\prime}(a)$ contains no separating triangle, then $a$ is a leaf of $T$. Otherwise, consider every maximal separating triangle $(u, v, z)$ of $G^{\prime}(a)$; then, insert a node $b$ in $T$ as a child of $a$, such that $G^{\prime}(b)$ is the subgraph of $G^{\prime}(a)$ induced by the vertices internal to or on the border of cycle $(u, v, z)$. For each node $a \in T$, denote as $V^{\prime}(a)$ and $E^{\prime}(a)$ the vertex set and the edge set of $G^{\prime}(a)$. Further, for each node $a \in T$, let $G(a)=(V(a), E(a))$ denote the subgraph of $G^{\prime}(a)$ induced by all the vertices which are not internal to any separating triangle of $G^{\prime}(a)$. Note that $G(a)$ is 4 -connected for every $a \in V^{\prime}$.

We now define a total ordering $o(V)$ of $V$ and we later prove that the maximum twist size of $o(V)$ is $O(f(n))$. Ordering $o(V)$ is constructed by induction on $T$. In the base case $a$ is a leaf; then let $o\left(V^{\prime}(a)\right)$ be any total ordering of $V^{\prime}(a)$ such that the maximum twist size of $o\left(V^{\prime}(a)\right)$ is $f(n)$. Such an ordering exists by hypothesis, since $G^{\prime}(a)$ is 4 -connected. In the inductive case, let $a_{1}, \ldots, a_{m}$ be the children of $a$ in $T$, 


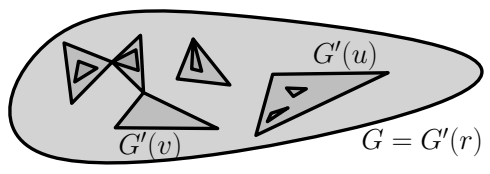

(a)

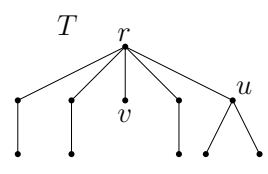

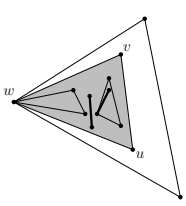

(b)

Fig. 1. (a) Tree $T$ capturing the structure of the separating triangles in $G$. (b) Graph $G^{\prime}(a)$; the thick edges belong to $M_{0}$.

where total orderings $o\left(V^{\prime}\left(a_{1}\right)\right), \ldots, o\left(V^{\prime}\left(a_{m}\right)\right)$ of $V^{\prime}\left(a_{1}\right), \ldots, V^{\prime}\left(a_{m}\right)$, respectively, have already been computed. Compute a total ordering $o(V(a))$ of $V(a)$ such that the maximum twist size of $o(V(a))$ is $f(n)$. Again, such an ordering exists by hypothesis, since $G(a)$ is 4-connected. Next, we merge $o\left(V^{\prime}\left(a_{1}\right)\right), \ldots, o\left(V^{\prime}\left(a_{m}\right)\right)$ with $o(V(a))$. In order to do this, we define the operation of merging an ordering $V_{2}$ into an ordering $V_{1}$, that takes as input two total vertex orderings $o\left(V_{1}\right)$ and $o\left(V_{2}\right)$ such that $V_{1}$ and $V_{2}$ share a single vertex $v$, and outputs a single total vertex ordering $o\left(V_{1} \cup V_{2}\right)$ of $V_{1} \cup V_{2}$ such that $o\left(V_{1} \cup V_{2}\right)$ coincides with $o\left(V_{i}\right)$ when restricted to the vertices in $V_{i}$, for $i=1,2$, and such that every vertex of $V_{1}$ that precedes $v$ in $o\left(V_{1}\right)$ (resp. follows $v$ in $o\left(V_{1}\right)$ ) precedes all the vertices of $V_{2}$ in $o(V)$ (resp. follows all the vertices of $V_{2}$ in $o(V)$ ). Denote by $b(H)$, by $m(H)$, and by $t(H)$ the bottom vertex, the middle vertex, and the top vertex of an upward triangulation $H$, respectively. Then, ordering $o\left(V^{\prime}(a)\right)$ is defined as follows: Let $o_{1}=o(V(a))$ and let $o_{i+1}$ be the ordering obtained by merging $o\left(V^{\prime}\left(a_{i}\right)\right) \backslash$ $\left\{b\left(G^{\prime}\left(a_{i}\right)\right), t\left(G^{\prime}\left(a_{i}\right)\right)\right\}$ into $o_{i}$, for $i=1, \ldots, m$; then $o\left(V^{\prime}(a)\right)=o_{m+1}$. Observe that $o\left(V^{\prime}(a)\right)$ is an upward vertex ordering because $o(V(a)), o\left(V^{\prime}\left(a_{1}\right)\right), \ldots, o\left(V^{\prime}\left(a_{m}\right)\right)$ are and because of the definition of the merging operation.

We now prove that the size of the maximum twist induced by $o(V)$ is $O(f(n))$. Let $M=\left\{e_{1}=\left(u_{1}, v_{1}\right), \ldots, e_{k}=\left(u_{k}, v_{k}\right)\right\}$ denote any maximal twist induced by $o(V)$. We have the following:

Claim 1. Let a be a node of T. Let $a_{1}$ and $a_{2}$ be two distinct children of a. There is no pair of distinct edges $\left(u_{i}, v_{i}\right),\left(u_{j}, v_{j}\right)$ in $M$ such that $\left(u_{i}, v_{i}\right) \in E^{\prime}\left(a_{1}\right),\left(u_{j}, v_{j}\right) \in$ $E^{\prime}\left(a_{2}\right)$, and $\left\{u_{i}, v_{i}, u_{j}, v_{j}\right\} \cap V(a)=\emptyset$.

Proof: Let $\left(u^{1}, v^{1}, z^{1}\right)$ and $\left(u^{2}, v^{2}, z^{2}\right)$ be the separating triangles of $G^{\prime}(a)$ that delimit the outer faces of $G^{\prime}\left(a_{1}\right)$ and $G^{\prime}\left(a_{2}\right)$, where $v^{i}$ is the middle vertex of $G^{\prime}\left(a_{i}\right)$, for $i=1,2$. If $v^{1} \neq v^{2}$, then, by the construction of $o(V)$, all internal vertices of $G^{\prime}\left(a_{1}\right)$ precede all internal vertices of $G^{\prime}\left(a_{2}\right)$ or vice versa, thus $e_{i}$ and $e_{j}$ do not both belong to $M$. Otherwise, $v^{1}=v^{2}$. Then, again by the construction of $o(V), e_{i}$ and $e_{j}$ are nested, thus they do not both belong to $M$.

Let $r$ be the root of $T$. We assume that $G$ is "minimal", that is, we assume that there exists no child $a$ of $r$ such that all the edges in $M$ belong to $G^{\prime}(a)$. Indeed, if such a child exists, graph $G=G^{\prime}(r)$ can be replaced by $G^{\prime}(a)$, and the bound on the size of $M$ can be achieved by arguing on $G^{\prime}(a)$ rather than on $G^{\prime}(r)$. Denote by $M_{i}$, with $i=0,1,2$, the subset of $M$ that contains all the edges having $i$ endpoints in $V(r)$. Observe that $|M|=\left|M_{0}\right|+\left|M_{1}\right|+\left|M_{2}\right|$, hence it suffices to prove that $\left|M_{i}\right| \in O(f(n))$, for 
$i=0,1,2$, in order to prove the theorem. By hypothesis and since $G(r)$ is 4-connected, we have $\left|M_{2}\right| \leq f(n)$. We now deal with the edges in $M_{1}$.

Claim 2. $\left|M_{1}\right| \in O(f(n))$.

Proof: First, we argue that $M_{1}$ contains at most one edge $e$ such that an end-vertex of $e$ is the middle vertex of an upward planar triangulation $G^{\prime}(a)$, for some child $a$ of $r$. Indeed, by the vertex ordering's construction, any two such edges, say $e_{a}$ and $e_{b}$, are either incident to the same vertex or are such that both end-vertices of $e_{a}$ come before both end-vertices of $e_{b}$ in $o\left(V^{\prime}(a)\right)$. Thus, it is enough to bound the number of edges in $M_{1}$ whose end-vertex in $V(r)$ is the bottom vertex or the top vertex of an upward planar triangulation $G^{\prime}(a)$, where $a$ is a child of $r$.

Let $M_{1}^{b}$ (resp. $M_{1}^{t}$ ) be the subset of the edges in $M_{1}$ whose end-vertex in $V(r)$ is the bottom vertex (resp. the top vertex) of an upward planar triangulation $G^{\prime}(a)$, where $a$ is a child of $r$. Observe, that by the above observation, $|M| \leq\left|M_{1}^{b}\right|+\left|M_{1}^{t}\right|+1$. In the following we bound $\left|M_{1}^{b}\right|$ (the bound for $\left|M_{1}^{t}\right|$ can be obtained analogously).

Consider any edge $(u, v) \in M_{1}^{b}$, where $u \in V(r)$. We define a corresponding edge of $(u, v)$ in $G(r)$ as follows. Let $a_{u, v}$ be the child of $r$ such that $G^{\prime}\left(a_{u, v}\right)$ contains edge $(u, v)$. Further, denote by $m_{u, v}$ the middle vertex of $G^{\prime}\left(a_{u, v}\right)$. Then, $\left(u, m_{u, v}\right)$ is the corresponding edge of $(u, v)$ in $G(r)$. Observe that edge $\left(u, m_{u, v}\right)$ exists and belongs to $E(r)$. Now consider the multi-set $E_{1}^{b}$ of the corresponding edges, that is $E_{1}^{b}=\left\{\left(u, m_{u, v}\right) \mid(u, v) \in M_{1}^{b}\right\}$. First, we have that, for each vertex $w$ in $V(r)$, there exist at most two edges $(z, w)$ in $E_{1}^{b}$, since each vertex in $V(r)$ is the middle vertex of at most two upward planar triangulations $G^{\prime}\left(a_{i}\right)$, where $a_{i}$ is a child of $r$, and since $G^{\prime}\left(a_{i}\right)$ has at most one edge in $M_{1}^{b}$. If there exist two edges $\left(z_{1}, w\right)$ and $\left(z_{2}, w\right)$ in $E_{1}^{b}$, then remove one of them. Then, after such deletions, $\left|E_{1}^{b}\right| \geq\left|M_{1}^{b}\right| / 2$.

Next, we prove that each vertex in $V(r)$ is an end-vertex of at most two edges in $E_{1}^{b}$. Namely, consider any two edges $\left(u_{1}, v_{1}\right)$ and $\left(u_{2}, v_{2}\right)$ in $E_{1}^{b}$. Then, $v_{1} \neq v_{2}$ because of the deletions performed on $E_{1}^{b}$, and $u_{1} \neq u_{2}$ as otherwise the corresponding edges in $M_{1}^{b}$ would share a vertex, contradicting the assumption that $M$ is a twist; thus, each vertex in $V(r)$ is the source of at most one edge in $E_{1}^{b}$ and the sink of at most one edge in $E_{1}^{b}$. Since the degree of graph $\left(V(r), E_{1}^{b}\right)$ is two, there exists a subset $E^{*}$ of $E_{1}^{b}$ such that the degree of graph $\left(V(r), E^{*}\right)$ is one and $\left|E^{*}\right| \geq\left|E_{1}^{b}\right| / 3$.

Finally, we have that every two edges in $E^{*}$ cross. Namely, if they do not, then by the vertex ordering's construction the corresponding edges in $M_{1}^{b}$ would not cross either, thus contradicting the assumption that $M$ is a twist.

Since $E^{*} \subseteq E(r)$ and the maximum size of a twist of edges in $E(r)$ is $f(n)$, given that $G(r)$ is 4-connected, it follows that $E^{*} \leq f(n)$. Using $\left|E^{*}\right| \geq\left|E_{1}^{b}\right| / 3$ and $\left|E_{1}^{b}\right| \geq$ $\left|M_{1}^{b}\right| / 2$, we get $\left|M_{1}^{b}\right| \leq 6 f(n)$. Such an inequality, together with the analogous bound $\left|M_{1}^{t}\right| \leq 6 f(n)$ and with $|M| \leq\left|M_{1}^{b}\right|+\left|M_{1}^{t}\right|+1$, proves the theorem.

We now proceed by bounding the size of $M_{0}$.

Claim 3. $\left|M_{0}\right| \in O(f(n))$.

Proof: By Claim 1, all the edges in $M_{0}$ belong to a graph $G^{\prime}(a)$, for a certain descendant $a$ of $r$. Let us choose $a$ so that the length of the path from $a$ to $r$ is maximized. Let $w$ be the middle vertex of the separating triangle $(u, v, w)$ delimiting $G^{\prime}(a)$. Let $a^{\prime}$ denote 
the child of $r$ which is an ancestor of $a$ or that coincides with $a$. Let $w^{\prime}$ be the middle vertex of the separating triangle $\left(u^{\prime}, v^{\prime}, w^{\prime}\right)$ delimiting $G^{\prime}\left(a^{\prime}\right)$.

For any edge $(y, z) \in M_{0}$, we have that $(y, z)$ "nests around $w^{\prime \prime}$, that is, $y$ precedes $w^{\prime}$ and $w^{\prime}$ precedes $z$ in $o(V)$. Indeed, if both $y$ and $z$ precede $w^{\prime}$ in $o(V)$ (or if they both follow $w^{\prime}$ in $o(V)$ ), then only the edges in $G^{\prime}\left(a^{\prime}\right)$ can possibly cross $(y, z)$, by the construction of $o(V)$, thus contradicting the minimality of $r$.

If $w \neq w^{\prime}$, then $\left|M_{0}\right| \leq 3$, since only the edges incident to $u, v$ and $w$ can belong to $M_{0}$. Otherwise we have $w^{\prime}=w$ (see Fig. 1(b)). Consider graph $G^{\prime}(a)$; partition the edges in $M_{0}$ into two subsets, namely $M_{0}^{\prime}$ contains all the edges of $M_{0}$ having at least one end-vertex in $V(a)$ and $M_{0}^{\prime \prime}$ contains all the edges of $M_{0}$ having no end-vertex in $V(a)$. By definition of $a$ and by Claim $1,\left|M_{0}^{\prime}\right|>0$, as otherwise there would exist a child of $a$ containing all the edges of $M_{0}$. However, by Claim 2 applied to $G^{\prime}(a)$ and by the hypothesis of the theorem, we have $\left|M_{0}^{\prime}\right| \in O(f(n))$. Moreover, every edge in $M_{0}^{\prime \prime}$ is in a separating triangle of $G^{\prime}(a)$ having $w$ as middle vertex; however, any such edge is nested inside any edge of $M_{0}^{\prime}$; thus, since $\left|M_{0}^{\prime}\right|>0$, we have $\left|M_{0}^{\prime \prime}\right|=0$ and hence $\left|M_{0}\right| \in O(f(n))$, which concludes the proof.

Since $\left|M_{i}\right| \in O(f(n))$, for $i=0,1,2$, it follows that $|M| \in O(f(n))$, thus proving Theorem[1, By Lemmata 1 and 2, we have the following:

Corollary 1. If every $n$-vertex upward planar 4-connected triangulation has $o\left(\frac{n}{\log n}\right)$ page number, then every $n$-vertex upward planar triangulation has o $n)$ page number.

Corollary 2. Every upward planar 3-tree has $O(1)$ page number.

\section{Page Number and Diameter}

In this section we study the relationship between the page number of an upward planar DAG and its diameter $D$. We show that upward planar DAGs with small diameter have sub-linear page number. Notice that such a result pairs the observation that graphs with diameter $n-o(n)$ have sub-linear page number as well, given that upward planar Hamiltonian DAGs have page number two. We have the following:

Theorem 2. Every n-vertex upward planar triangulation whose diameter is at most $D$ admits an upward vertex ordering whose maximum twist size $t(n)$ is a function satisfying $t(n) \leq a D+t\left(\frac{n}{2}\right)+b$, for some constants $a$ and $b$.

We will prove the statement for a family of upward planar DAGs that is strictly larger than the family of upward planar triangulations. Namely, we call upward cactus an embedded upward planar DAG $G$ having exactly one source $s(G)$ and such that every internal face is delimited by a 3-cycle. See Fig. 2. Observe that an upward planar triangulation is an upward cactus.

Consider an upward cactus $G$. We call monotone path any directed path $P=\left(u_{1}, \ldots, u_{k}\right)$ from $s(G)$ to a sink of $G$. Consider an upward planar drawing $\Gamma$ of $G$ in which $u_{k}$ is the vertex with highest $y$-coordinate. Observe that such a drawing $\Gamma$ always exists because $G$ is an upward cactus. Then, we define the left side of $P$ as the 


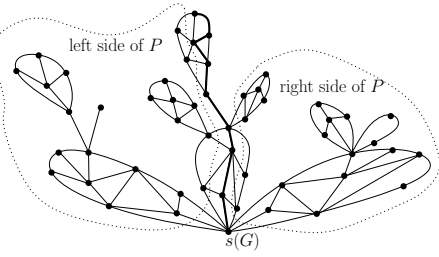

Fig. 2. An upward cactus $G$. The thick edges represent a monotone path $P$

subgraph of $G$ induced by all the vertices which are to the left of the Jordan curve representing $P$ in $\Gamma$. The right side of $P$ is defined analogously. Observe that the vertices of $P$, the vertices of the left side of $P$, and the vertices of the right side of $P$ form a partition of the vertices of $G$. We have the following:

Claim 4. In every $n$-vertex upward cactus there exists a monotone path $P$ such that both the left side of $P$ and the right side of $P$ have less than $\frac{n}{2}$ vertices.

We now prove the statement of the theorem for every $n$-vertex upward cactus $G$ with diameter at most $D$. The proof is by induction on $n$. If $n \leq 3$, then in any upward vertex ordering of $G$ the maximum twist size is 1 , hence $t(3) \leq b$, for any $b \geq 1$, thus proving the base case.

Suppose that $n>3$. By Claim 4 there exists a monotone path $P$ in $G$ such that both the left side of $P$ and the right side of $P$ have less than $\frac{n}{2}$ vertices. We now associate each vertex in the left side of $P$ and each vertex in the right side of $P$ to a vertex of $P$. Namely, we associate a vertex $v$ in the left side of $P$ to the vertex $u_{i}$ of $P$ such that there exists a directed path from $u_{i}$ to $v$ and such that, for every $j>i$, there exists no directed path from $u_{j}$ to $v$. Observe that, for every vertex $v$ in the left side of $P$, there exists a directed path from $s(G)$ to $v$, since $G$ has a unique source, hence $v$ is associated to exactly one vertex of $P$. Then, we call left bag of $u_{i}$ the set of vertices in the left side of $P$ which are associated to $u_{i}$, for each $i=1, \ldots, k$. Vertices in the right side of $P$ are associated to vertices of $P$ analogously, thus analogously defining the right bag of $u_{i}$, for each $i=1, \ldots, k$. We have the following:

Claim 5. The subgraph $G_{i}^{L}$ of $G$ induced by the left bag of $u_{i}$ and by $u_{i}$ is an upward cactus, for every $i=1, \ldots, k$.

An analogous claim holds for the subgraph $G_{i}^{R}$ of $G$ induced by the right bag of $u_{i}$ and by $u_{i}$.

Next, we construct an upward vertex ordering of $G$. This is done as follows. First, inductively construct an upward vertex ordering $\sigma_{i}^{L}$ of $G_{i}^{L}$ and an upward vertex ordering $\sigma_{i}^{R}$ of $G_{i}^{R}$, for $i=1, \ldots, k$, such that the maximum twist size of each of $\sigma_{i}^{R}$ and $\sigma_{i}^{L}$ is $t\left(\frac{n}{2}\right)$. This is possible since $G_{i}^{L}$ and $G_{i}^{R}$ are upward cacti, by Claim 5 , and they have less than $\frac{n}{2}$ vertices, by Claim 4, Observe that $u_{i}$ is the first vertex both in $\sigma_{i}^{L}$ and in $\sigma_{i}^{R}$, given that it is the only source of both $G_{i}^{L}$ and $G_{i}^{R}$. Then, denote by $\sigma_{i}$ the vertex ordering of $G_{i}^{L} \cup G_{i}^{R}$ which is obtained by concatenating $\sigma_{i}^{L}$ and $\sigma_{i}^{R} \backslash\left\{u_{i}\right\}$. Finally a vertex ordering $\sigma$ of $G$ is obtained by concatenating $\sigma_{1}, \sigma_{2}, \ldots, \sigma_{k}$. 
Claim 6. $\sigma$ is an upward vertex ordering.

Next, we prove that the maximum twist size $t(n)$ of $\sigma$ is at most $a D+t\left(\frac{n}{2}\right)+b$, for some constants $a$ and $b$.

First, observe that the edges that have both end-vertices in $P$ create twists of size at most two, since the graph induced by the vertices of $P$ is upward planar Hamiltonian.

Second, we discuss the size of a twist composed of intra-bag edges, which are edges whose both end-vertices are associated to the same vertex of $P$. Consider any edge $e_{i}^{L}$ of $G_{i}^{L}$ and any edge $e_{i}^{R}$ of $G_{i}^{R}$. Such edges do not cross. Namely, if such edges are both incident to $u_{i}$, then they do not cross by definition. If $e_{i}^{R}$ is not incident to $u_{i}$, then both end-vertices of $e_{i}^{R}$ come after both end-vertices of $e_{i}^{L}$, by construction, hence such edges do not cross. Moreover, if $e_{i}^{R}$ is incident to $u_{i}$ and $e_{i}^{L}$ is not, then $e_{i}^{L}$ is nested inside $e_{i}^{R}$, by construction, hence such edges do not cross. It follows that the maximum size of a twist of intra-bag edges is equal to the maximum twist size of $\sigma$ restricted to the vertices in $G_{i}^{a}$ for some $a \in\{L, R\}$ and some $1 \leq i \leq k$. By Claim 5, graph $G_{i}^{a}$ is an upward cactus. Moreover, by Claim $4 G_{i}^{a}$ has at most $\frac{n}{2}$ vertices, hence the maximum size of a twist of intra-bag edges is at most $t\left(\frac{n}{2}\right)$.

Third, we discuss the maximum size of a twist composed of inter-bag edges, which are edges whose end-vertices are associated to distinct vertices of $P$. We show that the maximum size of a twist composed of inter-bag edges in the left side of $P$ is $2 D$. An analogous proof shows that the maximum size of a twist composed of inter-bag edges in the right side of $P$ is also $2 D$.

Consider any two inter-bag edges $\left(w_{1}, w_{2}\right)$ and $\left(w_{3}, w_{4}\right)$ in the left side of $P$. Suppose that $\left(w_{1}, w_{2}\right)$ and $\left(w_{3}, w_{4}\right)$ cross in $\sigma$. Denote by $u_{j_{1}}, u_{j_{2}}, u_{j_{3}}$, and $u_{j_{4}}$, such that $u_{j_{1}}<u_{j_{2}}$ and $u_{j_{3}}<u_{j_{4}}$, the vertices of $P$ vertices $w_{1}, w_{2}, w_{3}$, and $w_{4}$ have been assigned to, respectively. The following claim asserts that any two inter-bag edges $\left(w_{1}, w_{2}\right)$ and $\left(w_{3}, w_{4}\right)$ that cross in $\sigma$ either have their sources assigned to the same vertex of $P$, or have their destinations assigned to the same vertex of $P$, or the source of one of them and the destination of the other of them are assigned to the same vertex of $P$.

Claim 7. At least one of the following holds: $j_{1}=j_{3}<j_{2}, j_{4}$, or $j_{1}<j_{2}=j_{3}<j_{4}$, or $j_{3}<j_{4}=j_{1}<j_{2}$, or $j_{1}, j_{3}<j_{2}=j_{4}$.

Hence, if there are more than $2 D$ inter-bag edges pairwise crossing in the left side of $P$, then either there are more than $D$ inter-bag edges pairwise crossing in the left side of $P$ such that the origins of such edges have all been assigned to the same vertex of $P$, or there are more than $D$ inter-bag edges pairwise crossing in the left side of $P$ such that the destinations of such edges have all been assigned to the same vertex of $P$. In the following, we discuss such two cases.

Claim 8. Suppose that $G$ contains inter-bag edges $\left(v_{1}, w_{1}\right),\left(v_{2}, w_{2}\right), \ldots,\left(v_{k}, w_{k}\right)$ in the left side of $P$, where $v_{1}<_{\sigma} v_{2}<_{\sigma} \cdots<_{\sigma} v_{k}<_{\sigma} w_{1}<_{\sigma} w_{2}<_{\sigma} \cdots<_{\sigma} w_{k}$ and where all the vertices $w_{i}$ have been assigned to the same vertex $u_{l}$ of $P$, for $i=1, \ldots, k$, or all the vertices $v_{i}$ have been assigned to the same vertex $u_{l}$ of $P$, for $i=1, \ldots, k$. Then, there exists a directed path starting at $u_{l}$ and passing through $w_{1}, w_{2}, \ldots, w_{k}$. 
Since by hypothesis any directed path contains at most $D$ vertices, then, by Claim 8 . the maximum size of a twist of inter-bag edges sharing their destinations in the left side of $P$ is at most $D$ and the maximum size of a twist of inter-bag edges sharing their origins in the left side of $P$ is at most $D$. Hence, by Claim 7 the maximum size of a twist of inter-bag edges in the left side of $P$ is at most $2 D$ and the maximum size of a twist of inter-bag edges is at most $4 D$. Since every edge of $G$ is either an edge having both end-vertices in $P$, or is an intra-bag edge, or is an inter-bag edge, it follows that the maximum size of a twist in $\sigma$ is $t(n)=2+t\left(\frac{n}{2}\right)+4 D$, thus proving Theorem 2 .

By Lemma11 we have the following:

Corollary 3. Every n-vertex upward planar triangulation whose diameter is $o\left(\frac{n}{\log n}\right)$ has o(n) page number.

\section{Page Number and Degree}

In this section we discuss the relationship between the page number of a graph and its degree. We prove the following theorem.

Theorem 3. Let $f(n)$ be any function such that $f(n) \in \Omega(\sqrt{n})$ and $f(n) \in O(n)$. Suppose that every $n$-vertex upward planar triangulation whose degree is $O(f(n))$ admits a book embedding with $O(g(n))$ pages, for some function $g(n) \in \Omega(1)$ and $g(n) \in O(n)$. Then, every $n$-vertex upward planar triangulation admits a book embedding with $O\left(g(n)+\frac{n}{f(n)}\right)$ pages.

Consider any $n$-vertex upward planar triangulation $G$. We transform $G$ into an $O(n)$ vertex upward planar triangulation $G^{\prime}$ with degree $O(f(n))$ as follows. Fix any constant $c>0$ and denote by $u_{1}, \ldots, u_{k}$ any ordering of the vertices of $G$ whose degree is greater than $c f(n)$.

For $i=1, \ldots, k$, consider vertex $u_{i}$. Suppose that $u_{i}$ is an internal vertex of $G$, the case in which $u_{i}$ is an external vertex being analogous. Since it is an upward planar triangulation, $G$ has exactly two faces $\left(v_{1}, v_{2}, u_{i}\right)$ and $\left(v_{3}, v_{4}, u_{i}\right)$ incident to $u_{i}$ such that edges $\left(v_{1}, u_{i}\right)$ and $\left(v_{4}, u_{i}\right)$ are incoming $u_{i}$ and such that edges $\left(u_{i}, v_{2}\right)$ and $\left(u_{i}, v_{3}\right)$ are outgoing $u_{i}$. Assume, w.l.o.g., that $\left(v_{1}, u_{i}\right),\left(u_{i}, v_{2}\right),\left(u_{i}, v_{3}\right)$, and $\left(v_{4}, u_{i}\right)$ appear in this clockwise order around $u_{i}$. Denote by $w_{1}=v_{2}, w_{2}, \ldots, w_{x-1}, w_{x}=$ $v_{3}, w_{1}^{\prime}=v_{4}, w_{2}^{\prime}, \ldots, w_{y-1}^{\prime}, w_{y}^{\prime}=v_{1}$ the clockwise order of the neighbors of $u_{i}$ (see Fig. $3\left(\right.$ a)). Remove $u_{i}$ and its incident edges from $G$. Let $M=\left\lceil\frac{x}{f(n)-1}\right\rceil$ and $N=\left\lceil\frac{y}{f(n)-1}\right\rceil$. Insert $M+N+2$ vertices $z_{1}, \ldots, z_{M+N+2}$ in $G$ inside the cycle of the neighbors of $u_{i}$. Insert an edge from $z_{j}$ to $z_{j+1}$, for $j=1, \ldots, M$, insert an edge from $z_{j+1}$ to $z_{j}$, for $j=M+1, \ldots, M+N+1$, and insert edges from $z_{M+2}$ to $z_{1}, \ldots, z_{M}$ and from $z_{M+3}, \ldots, z_{M+N+2}$ to $z_{1}$. Insert edges from $v_{1}$ to $z_{1}$, from $z_{1}$ to $v_{2}$, from $v_{4}$ to $z_{M+2}$, and from $z_{M+2}$ to $v_{3}$. Insert edges from $z_{j}$ to $w_{(j-2)(f(n)-1)+1}, w_{(j-2)(f(n)-1)+2}, \ldots, w_{(j-1)(f(n)-1)}$, for $j=2, \ldots, M+1$; insert edges from $w_{(j-2)(f(n)-1)+1}^{\prime}, w_{(j-2)(f(n)-1)+2}^{\prime}, \ldots, w_{(j-1)(f(n)-1)}^{\prime}$ to $z_{M+j}$, for $j=3, \ldots, N+2$. See Fig. 3 (b).

It is easy to see that the triangulation $G^{\prime}$ obtained from $G$ after all vertices $u_{1}, \ldots, u_{k}$ have been considered is upward planar. We have the following. 


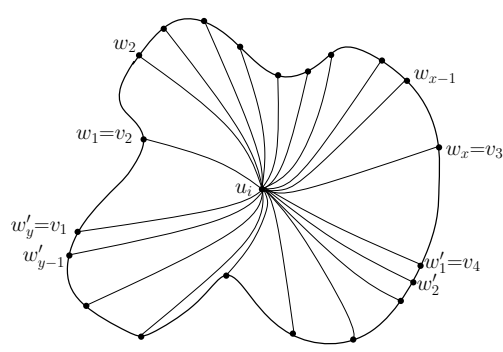

(a)

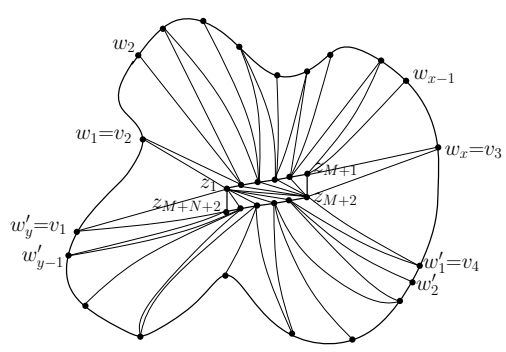

(b)

Fig. 3. (a) Neighbors of a high-degree vertex $u_{i}$. (b) Replacing $u_{i}$ with lower-degree vertices, assuming $f(n)=3$.

Claim 9. $G^{\prime}$ has $O(n)$ vertices and $O(f(n))$ degree. Moreover, for every upward vertex ordering $\sigma^{\prime}$ of $G^{\prime}$, there exists an upward vertex ordering $\sigma$ of $G$ such that $\sigma$ and $\sigma^{\prime}$ restricted to the vertices that are both in $G$ and in $G^{\prime}$ coincide.

We now describe how to compute a book embedding of $G$ in $O\left(g(n)+\frac{n}{f(n)}\right)$ pages. First, construct the upward planar triangulation $G^{\prime}$ as above. Second, construct a book embedding of $G^{\prime}$ into $O(g(n))$ pages. Such a book embedding exists by hypothesis, since $G^{\prime}$ has $O(n)$ vertices and $O(f(n))$ degree (by Claim 9). Denote by $\sigma^{\prime}$ the total ordering of the vertices of $G^{\prime}$ in the constructed book embedding. Construct any total ordering $\sigma$ of the vertices of $G$ such that $\sigma$ and $\sigma^{\prime}$ restricted to the vertices that are both in $G$ and in $G^{\prime}$ coincide. Such an ordering exists (and can be easily constructed) by Claim 9. The edges of $G$ can be assigned to pages as follows: $O(g(n))$ pages suffice to accommodate all the edges that are both in $G$ and in $G^{\prime}$; moreover, one page can be used to accommodate all the edges incident to vertex $u_{i}$, for $i=1, \ldots, k \in O\left(\frac{n}{f(n)}\right)$. It follows that $G$ has a book embedding in $O\left(g(n)+\frac{n}{f(n)}\right)$ pages, thus proving Theorem 3

Corollary 4. Every n-vertex upward planar triangulation has o $(n)$ page number if and only if every $n$-vertex upward planar triangulation with degree $O(\sqrt{n})$ has o(n) page number.

\section{Conclusions}

In this paper we studied the relationship between the page number of an upward planar triangulation $G$ and three important parameters of $G$ : The connectivity, the diameter, and the degree. It would be interesting, in our opinion, to understand whether the statements of Theorems 1 and 2 can be referred to the page number rather than to the maximum twist size. That is: (1) Is it true that any upward planar triangulation $G$ has page number $O(k)$ if and only if every maximal 4-connected subgraph of $G$ has page number $O(k)$ ? (2) Is it true that any $n$-vertex upward planar triangulation $G$ with diameter $D$ has page number $p(n)$ satisfying $p(n)=p\left(\frac{n}{2}\right)+a D+b$, for some constants $a$ and $b$ ?

Determining whether every $n$-vertex upward planar DAG has $o(n)$ page number and whether there exist upward planar DAGs with $\omega(1)$ page number remain among the most important problems in the theory of linear graph layouts. 
Acknowledgments. The first author would like to thank Patrizio Angelini, Giuseppe Di Battista, and Stefano Saraulli for very useful discussions.

\section{References}

1. Alzohairi, M., Rival, I.: Series-Parallel Planar Ordered Sets Have Pagenumber Two. In: North, S.C. (ed.) GD 1996. LNCS, vol. 1190, pp. 11-24. Springer, Heidelberg (1997)

2. Buss, J.F., Shor, P.W.: On the pagenumber of planar graphs. In: Symposium on Theory of Computing (STOC 1984), pp. 98-100. ACM (1984)

3. Cerný, J.: Coloring circle graphs. Elec. Notes Discr. Math. 29, 457-461 (2007)

4. Chung, F.R.K., Leighton, F.T., Rosenberg, A.L.: Embedding graphs in books: A layout problem with applications to VLSI design. SIAM J. Alg. Discr. Meth. 8, 33-58 (1987)

5. Di Battista, G., Tamassia, R.: Algorithms for plane representations of acyclic digraphs. Theor. Comp. Sci. 61, 175-198 (1988)

6. Di Giacomo, E., Didimo, W., Liotta, G., Wismath, S.K.: Book embeddability of seriesparallel digraphs. Algorithmica 45(4), 531-547 (2006)

7. Enomoto, H., Nakamigawa, T., Ota, K.: On the pagenumber of complete bipartite graphs. J. Comb. Th. Ser. B 71(1), 111-120 (1997)

8. Ganley, J.L., Heath, L.S.: The pagenumber of $k$-trees is $O(k)$. Discr. Appl. Math. 109(3), 215-221 (2001)

9. Heath, L.S.: Embedding planar graphs in seven pages. In: Foundations of Computer Science (FOCS 1984), pp. 74-83. IEEE (1984)

10. Heath, L.S., Istrail, S.: The pagenumber of genus $g$ graphs is $O(g)$. J. ACM 39(3), 479-501 (1992)

11. Heath, L.S., Leighton, F.T., Rosenberg, A.L.: Comparing queues and stacks as mechanisms for laying out graphs. SIAM J. Discr. Math. 5(3), 398-412 (1992)

12. Heath, L.S., Pemmaraju, S.V.: Stack and queue layouts of posets. SIAM J. Discr. Math. 10(4), 599-625 (1997)

13. Heath, L.S., Pemmaraju, S.V.: Stack and queue layouts of directed acyclic graphs: Part II. SIAM J. Computing 28(5), 1588-1626 (1999)

14. Heath, L.S., Pemmaraju, S.V., Trenk, A.N.: Stack and queue layouts of directed acyclic graphs: Part I. SIAM J. Computing 28(4), 1510-1539 (1999)

15. Kainen, P.C.: Thickness and coarseness of graphs. Abh. Math. Sem. Univ. Hamburg 39, 8895 (1973)

16. Kostochka, A.V., Kratochvíl, J.: Covering and coloring polygon-circle graphs. Discr. Math. 163(1-3), 299-305 (1997)

17. Malitz, S.M.: Genus $g$ graphs have pagenumber $O(\sqrt{g})$. J. Algorithms 17(1), 85-109 (1994)

18. Malitz, S.M.: Graphs with $e$ edges have pagenumber $O(\sqrt{e})$. J. Algorithms 17(1), 71-84 (1994)

19. Ollmann, L.T.: On the book thicknesses of various graphs. In: Hoffman, F., Levow, R.B., Thomas, R.S.D. (eds.) Southeastern Conference on Combinatorics, Graph Theory and Computing. Congressus Numerantium, vol. VIII, p. 459 (1973)

20. Rosenberg: The Diogenes approach to testable fault-tolerant arrays of processors. IEEE Trans. Comp. C-32, 902-910 (1983)

21. Tarjan, R.E.: Sorting using networks of queues and stacks. J. ACM 19(2), 341-346 (1972)

22. Yannakakis, M.: Embedding planar graphs in four pages. J. Comp. Syst. Sci. 38(1), 36-67 (1989) 